\title{
Política educativa, violencia y convivencia escolar. La experiencia en dos escuelas*
}

Adriana Carro Olvera ${ }^{a}$ José Alfonso Lima Gutiérrez ${ }^{b}$

\section{Resumen}

Ante los resultados poco efectivos que demostró el enfoque correctivo para atender el fenómeno de la violencia, aparecieron propuestas orientadas a la prevención, demostrando que es mejor anticiparse a las conductas antisociales que corregirlas. En ese marco, el tema de la convivencia escolar se ha inscrito como una herramienta de trabajo muy acogida desde la academia y en el plano institucional en México. De esta manera, el objetivo de la investigación fue identificar la política educativa orientada al desarrollo de la convivencia en dos escuelas en contextos diferenciados de nivel primaria del estado de Tlaxcala, México. El método de trabajo comprendió dos etapas: 1) el diagnóstico de la situación de la violencia escolar; y, 2) la identificación de la implementación de la política para la convivencia escolar. Los resultados demuestran situaciones de violencia y estrategias implementadas relativamente contrastantes donde se identifica a los docentes y directivos como los actores vertebrales de la política educativa para la convivencia escolar.

Palabras clave: Política educativa. Convivencia escolar. Escuela. Inclusión.

\section{Introducción}

México es un país marcado por la violencia. Los índices delictivos son cada vez mayores y se propagan en todas las regiones y espacios sociales sin excepción. Aunado a ello, y como un problema y consecuencia a la vez, está el hecho de que la gente ha normalizado el fenómeno, lo cual es sumamente preocupante ya que esta denominación surge cuando la sociedad lo percibe como parte de

\footnotetext{
* Proyecto de investigación financiado por el Fondo Sectorial de Investigación para la Educación SEP-CONACYT.

a Universidad Autónoma de Tlaxcala, Tlaxcala de Xicohténcatl ,Tlaxcala, México.

b Universidad Autónoma de Tlaxcala, Tlaxcala de Xicohténcatl ,Tlaxcala, México.
} 
su contexto cotidiano. Aunque se reconoce que las escuelas nunca han estado exentas de violencia, actualmente han registrado una tendencia incremental en las cifras, reflejo de la violencia estructural y cultural del país.

Desde hace algunas décadas, los enfoques preventivos fueron demostrando que si bien su complejidad es mayor y sus alcances son de largo plazo, los resultados son mejores ya que fortalecen los mecanismos formativos a partir de la hipótesis que la mejor alternativa para evitar las conductas antisociales es impedir que aparezcan o se reproduzcan. Como estrategia específica de prevención, las políticas de convivencia han constatado ser un instrumento oportuno y eficaz.

La convivencia como concepto y asunto público se incorporó en la agenda nacional desde el gobierno de Ernesto Zedillo (1994-2000), pero la convivencia escolar como tema de política educativa es posterior, ya que se integró de manera formal hasta el gobierno de Felipe Calderón (MÉXICO, 2008). De esta manera, en los últimos años ha sido permanente el interés de atender la violencia escolar mediante programas institucionales de convivencia y de estrategias pedagógicas transversales.

En la parte académica, investigaciones han establecido como una variable indispensable para la calidad educativa el clima emocional favorable al aprendizaje (ARON; MILICIC, 2013; CASTRO-PÉREZ; MORALES-RAMIREZ, 2015; GIRALDO; MENA, 2000). El estudio de la convivencia escolar es reciente, y como producto de políticas educativas también es escaso. Inició con el análisis de los fenómenos de violencia en los centros educativos (COLOMBO, 2011; DE LA CONCEPCIÓN, 2015; FUNK, 1997; RODNEY; GARCÍA, 2014), fijando la atención en el acoso escolar. En México, se presentó por primera vez como área temática en el tercer estado del conocimiento (1992-2002) del Consejo Mexicano de Investigación Educativa (Comie) (FIERRO; CARBAJAL; MARTÍNEZPARENTE, 2014). La convivencia como perspectiva de análisis incorporó aportes desde diversos acercamientos como: Educación Ciudadana, Educación En Valores, Educación Cívica Y Ética, Educación Inclusiva, cultura de la legalidad, Educación Para La Paz Y Los Derechos Humanos y Educación Para La Democracia (FIERRO; CARBAJAL; MARTÍNEZ-PARENTE, 2014).

La convivencia, en general, es entendida como el vivir unos con otros con base en determinadas relaciones sociales y en unos códigos valorativos, forzosamente subjetivos, en el marco de un contexto social determinado (JARES, 1999; SAUCEDO; MEJÍA 2013). La convivencia en el ámbito escolar no varía de este principio, de reciprocidad y puesta en práctica de valores democráticos (FIERRO et al., 2013; FURLÁN; SAUCEDO; LARA, 2004; HIRMAS; 
EROLES, 2008). Es la calidad de las relaciones interpersonales que dan lugar a un determinado clima escolar (ARARTEKO, 2006; LIRA; GOMES, 2018). Así entonces, aprender a convivir se ha convertido en una prioridad de la Educación; sin embargo, no obstante que su utilización es más frecuente en la actualidad, es considerada aún una categoría incipiente por consolidarse.

Las contribuciones aluden que el desarrollo de una convivencia escolar sana y pacífica se logra a través de procesos de aprendizaje democráticos e inclusivos, lo que significa que no son actos reflejos, ni respuestas automáticas (FIERRO; CARBAJAL; MARTÍNEZ-PARENTE, 2014; MILCÍADES, 2015). Conseguir un ambiente favorable para la convivencia va ligado a formas de gestión específicas que permiten abrir la lente para dejar de enfocar la violencia directa como el asunto central de la Educación e incorporar en escena la convivencia escolar (FIERRO et al., 2013).

Adicionalmente, la convivencia escolar tiene relevancia en la creación de políticas educativas sobre calidad por las implicaciones que la Educación tiene en la sociedad, "el relacionarse con otros en paz es el fundamento de una convivencia social democrática, la cual se constituye en un aprendizaje que debe ser intencionado desde las prácticas pedagógicas" (SANDOVAL MANRIQUEZ, 2014, p. 161).

Las actuales orientaciones normativas parten del fortalecimiento de una cultura organizativa escolar que permita la gestión de la convivencia. Es así como el objetivo de la investigación fue identificar la implementación de las políticas educativas orientadas al desarrollo de la convivencia en dos escuelas de tiempo completo de nivel primaria en contextos diferenciados, uno rural y otro urbano del Estado de Tlaxcala, México, a partir de un diagnóstico de violencia escolar.

\section{Las políticas educativas para la convivencia escolar}

Lograr que las sociedades sean más justas, inclusivas y equitativas es uno de los retos que enfrentan los sistemas educativos en el mundo; de ahí, que este postulado se estableciera como uno de los objetivos de los países miembros en la firma de la Agenda del Desarrollo Sostenible 2030, cuyo antecedente de referencia es lo dispuesto por la Declaración Universal de Derechos Humanos, proclamada en la sede de la Asamblea General de la Naciones Unidas ONU, el 10 de diciembre de 1948; la declaración en su artículo 26 señala que toda persona tiene derecho a la Educación, la cual tendrá por objeto, entre otras cosas, el respeto a los derechos humanos y a las libertades, y con ella se estaría en posibilidades de favorecer la comprensión, la tolerancia y la amistad entre las naciones y los grupos para alcanzar la paz (ONU, 2018). 
En México los antecedentes en la materia pueden remontarse hasta 1992, cuando la reforma al artículo tercero constitucional estableció que "la Educación contribuirá a una mejor convivencia humana, tanto por los elementos que aporte a fin de robustecer en el educando, junto con el aprecio para la dignidad de la persona como a la integridad de la familia..." (MÉXICO, 1992, párr. 5); sin embargo, el enunciado no se materializó en política pública del sector educativo o de alguna dependencia del ramo.

Las iniciativas de principios de siglo incorporaron el fomento de una cultura de no violencia con la inclusión del concepto de igualdad para orientar el respeto a los derechos humanos (MÉXICO, 2008). De esta premisa surgió el Programa de Escuela Segura (PES) en el año de 2007. El programa perduró cinco años y se focalizó en escuelas de mayor riesgo incluyendo todos los niveles y modalidades educativas del país, mediante una agenda de convivencia y seguridad escolar que permitiera reducir la violencia (MÉXICO, 2012a).

En el marco de la reforma educativa del año 2013 en México, la Secretaría de Educación Pública (SEP), con el fin de elevar la calidad de la Educación, promovió el denominado Sistema Básico de Mejora (SBM), asumiendo dentro de sus prioridades el desarrollo de una buena convivencia escolar para generar ambientes pacíficos, sanos, y formativos (MÉXICO, 2013a). Esto significa que el Estado mexicano la reconoce como un elemento para una Educación de calidad.

El artículo tercero constitucional se modificó como resultado de la reforma y postuló que "El estado garantizará la calidad en la Educación obligatoria de manera que los materiales y métodos educativos, la organización escolar, la infraestructura educativa y la idoneidad de los docentes y los directivos garanticen el máximo logro de aprendizaje de los alumnos [...] (Const., 2014, art. 3, párr. 3) [...] además contribuirá a la mejor convivencia humana”. Este precepto se traduce en el contenido de la Ley General de Educación (MÉXICO, 2014a) que refuerza la orientación de la Educación para contribuir a la mejor convivencia humana. A su vez, esta responsabilidad legal se incorporó en los objetivos institucionales del primer y tercer eje rector del Plan Nacional de Desarrollo 2013-2018 (MÉXICO, 2013b) para lograr un México en Paz y un México con Educación de Calidad. Por otro lado, el Programa Sectorial de Educación 2013-2018 determinó promover la formación docente sensible al respeto de los derechos humanos y la no violencia; y el acuerdo 717 (MÉXICO, 2014b) estableció como una de sus prioridades educativas el desarrollo de una buena convivencia escolar. 
Para alcanzar estos objetivos, el gobierno federal, a través de la Subsecretaría de Educación Básica de la SEP, implementó políticas y programas para reforzar las prioridades establecidas en el SBM, se enriqueció el Programa de Escuelas de Tiempo Completo (PETC), los Consejos Técnicos Escolares (CTE), las orientaciones para elaborar la Ruta de Mejora Escolar (RME) y el establecimiento del Programa Nacional de Convivencia Escolar (PNCE).

Así entonces, se constituyó una Política Nacional para la Convivencia Escolar con el propósito de favorecer en las escuelas el establecimiento de ambientes de convivencia armónica y pacífica que coadyuven a prevenir situaciones de acoso escolar y que propicien condiciones para mejorar los aprendizajes. Las escuelas públicas de Educación básica participantes reciben asistencia técnica pedagógica (MÉXICO, 2016). Aunado a ello, el PETC que apareció desde el año 2009, para el año 2013 se consolidó en cuanto a cobertura y suficiencia presupuestal, mediante la ampliación de la jornada escolar capitalizando el tiempo para el desarrollo académico, deportivo y cultural. Este programa prevé en una de sus líneas de trabajo transversal el aprender a convivir mediante un fichero de actividades didácticas (MÉXICO, 2013c).

Como se observa, desde hace algunos años se han diseñado sobre el tema estrategias conjuntas de diversos programas institucionales de la SEP. Con base en la literatura referida, se reconoce que los principios y orientaciones sobre la convivencia escolar van más allá de la lucha contra la violencia; sin embargo, para fines de investigación se revisa la situación de violencia y las acciones que promueven la convivencia en el marco de los programas de política educativa.

\section{Metodología}

El universo de estudio comprendió a directivos, docentes, padres de familia y alumnos. La selección de los centros escolares de Educación primaria fue a partir de tres criterios: 1. Que pertenecieran a contextos diferenciados, rural y urbano; 2. Que fueran escuelas de tiempo completo, situación que las obliga a instrumentar los diferentes programas de convivencia escolar de la SEP; y, 3. Que se encontraran localizadas en los municipios identificados como polígonos de mayor delincuencia en el estado de Tlaxcala ${ }^{1}$.

La Secretaría de Gobernación a través de la Ley General de Prevención Social de la Violencia y la Delincuencia (MÉXICO, 2012b) y del Programa Nacional de Prevención Social de la Violencia y la Delincuencia (Pronapred) que materializó la ley a partir del año 2013, elaboró un catálogo de los municipios con mayor índice de violencia en el país y en cada una de las entidades federativas. En el caso de Tlaxcala, durante los cuatro años que tuvo vigencia el programa, los municipios considerados (polígonos) fueron Calpulalpan y Tlaxcala (municipio capital del estado). 
El método para la obtención de la información fue mixto, cuantitativo para la primera etapa y cualitativo para la segunda. Los instrumentos cuantitativos se emplearon para elaborar un diagnóstico e identificar la intensidad de violencia, y los cualitativos para conocer la implementación de la política educativa de convivencia.

Primera etapa: Diagnóstico de la situación de violencia escolar. Este se realizó mediante la aplicación de cuestionarios a 145 alumnos de $4^{\circ}, 5^{\circ}$ y $6^{\circ}$ grado de ambas escuelas ( 86 de la escuela urbana y 59 de la escuela rural), de edades entre 9 y 13 años. El instrumento fue el Cuestionario de Violencia Escolar en Educación Primaria (Cuve-EP) elaborado por la División de Investigación y Estudios del Grupo ALBOR-COHS (ÁLVAREZ-GARCÍA; NÚÑEZ; DOBARRO, 2015). El instrumento consta de 34 ítems, con una escala de Likert de cinco opciones de respuesta que incluyen las dimensiones de nunca, pocas veces, algunas veces, muchas veces y siempre en sus siete factores del modelo (Ver Tabla 1).

Segunda etapa: Identificación de la implementación de la política educativa. Esta etapa de la investigación estuvo orientada a la prevención de la violencia, seguridad y convivencia escolar. Para obtener la información, se aplicaron 16 entrevistas a docentes de los mismos grados de $4^{\circ}, 5^{\circ}$ y $6^{\circ}$ de cada escuela, así como a cada uno de los directores. Para complementar la información, se entrevistaron a dos madres o padres de familia de alguno de los alumnos que participaron en el cuestionario.

En la interpretación de los datos se adoptó el esquema propuesto por Huberman y Miles (1994) y Rada (2007), que comienza con la tarea de reducción de datos, decodificación de la información, agrupamientos mediante las categorías de análisis, diseño de matrices factuales e inferenciales y, finalmente, elaboración del reporte de caso. Las unidades y categorías de análisis para conocer la implementación de la política se estructuraron a través de las líneas institucionales vigentes, además de los aspectos que inciden para su desempeño. Estos fueron:

1) El contexto: donde se describen los principales aspectos de cada una de las escuelas como ubicación, matrícula y características de la localidad y actividades preponderantes.

2) Las Prioridades del Sistema Básico de Mejora Escolar: Este apartado es el principal instrumento de la política educativa actual que se implementó a partir del gobierno federal en el acuerdo 717 (MÉXICO, 2014b) y comprende: a) mejora de las competencias de lectura, escritura y matemáticas; b) normalidad mínima de operación escolar; c) disminución del rezago y abandono escolar; y, d) desarrollo de una buena convivencia escolar. 
3) Funcionamiento como Escuela de Tiempo Completo: Se consideraron los elementos de la modalidad educativa como el cumplimiento de la ampliación de horario de manera eficiente bajo los principios rectores del programa "favorecer la Educación inclusiva y eliminar o minimizar las barreras que interfieren en el aprendizaje de los alumnos; donde además se fomente los ambientes seguros" (MÉXICO, 2013c, p. 9) bajo la implementación de las fichas didácticas específicas de trabajo, entre ellas la de aprender a convivir (MÉXICO, 2014c); la opinión de los padres de familia sobre la ampliación de jornada; $y$, el funcionamiento del comedor escolar.

5) Organización y funcionamiento del Consejo Técnico Escolar: Parte medular, ya que es el órgano colegiado de mayor decisión técnico pedagógica de cada escuela, y se encarga de tomar y ejecutar decisiones enfocadas a alcanzar el máximo logro de los aprendizajes de los alumnos. Está integrado por el director y todos los docentes, con la finalidad de identificar las problemáticas de los centros escolares y tomar las mejores decisiones para la ruta de mejora escolar (MÉXICO, 2017).

4) La Ruta de Mejora Escolar: Como planteamiento dinámico de gestión de mejora que conlleva un proceso que comprende el ejercicio de planeación a partir de la elaboración del diagnóstico, objetivos, metas e indicadores, además de la implementación, seguimiento, evaluación y rendición de cuentas (MÉXICO, 2014c). Toma como referentes para su elaboración las cuatro prioridades del SBM.

6) Uso y promoción de los materiales educativos: Cuya intención es la de identificar el uso y utilidad de los diversos materiales difundidos sobre el tema, como la Biblioteca de Gestión para la Convivencia Escolar, los manuales del PNCE para alumnos, docentes y padres de familia, así como el fichero y sus líneas específicas de trabajo orientadas para el programa de escuelas de tiempo completo, entre otros.

\section{Resultados}

\subsection{Diagnóstico de la violencia escolar en las escuelas}

Se presenta la información de los siete factores. Por razones de análisis e interpretación, la escala se concentró en tres aspectos de las cinco opciones posibles: pocas y algunas veces (baja intensidad de violencia); y, muchas veces y siempre (alta instensidad de violencia); la única que permanece sin cambios es la opción nunca. 


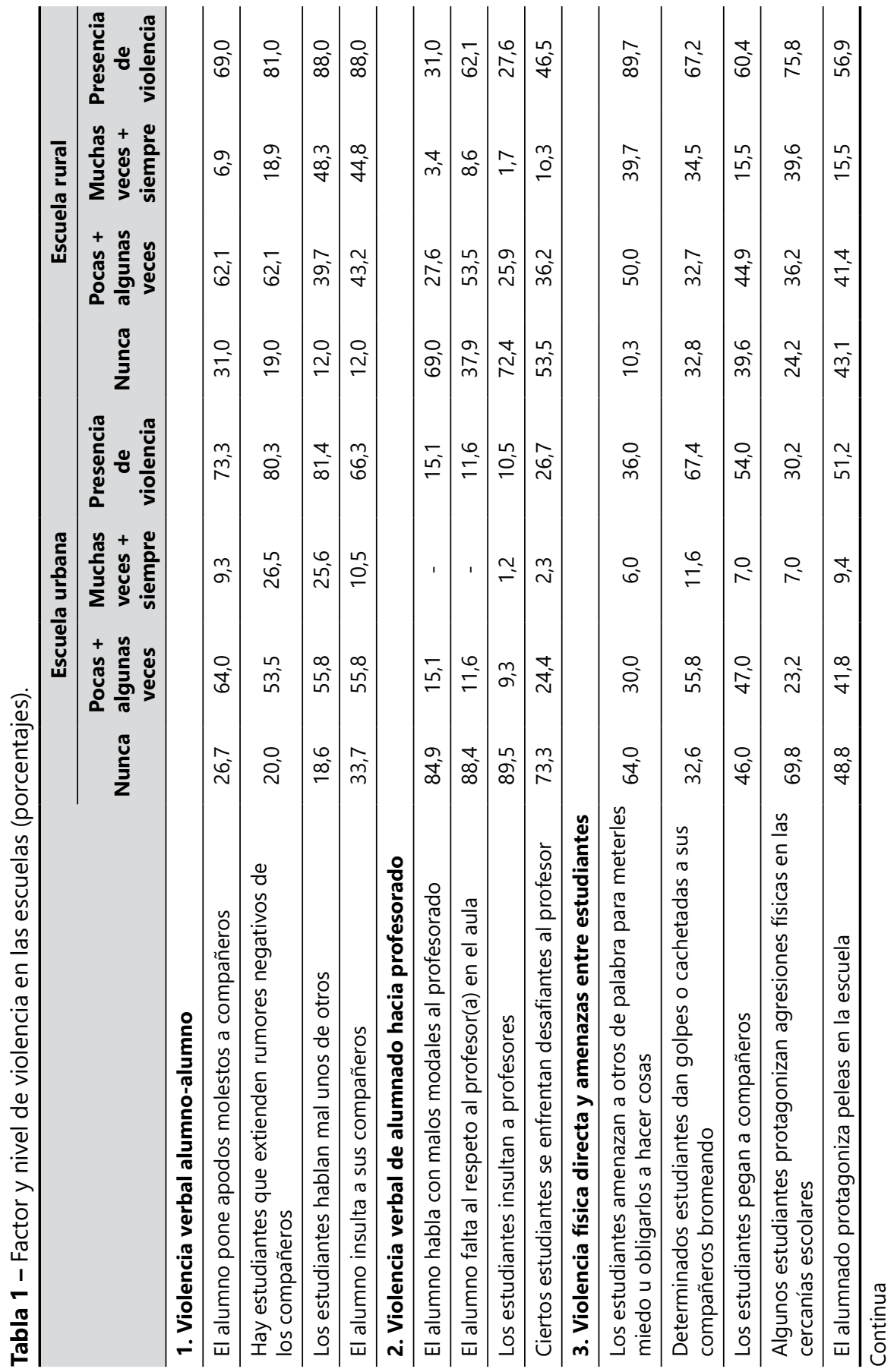




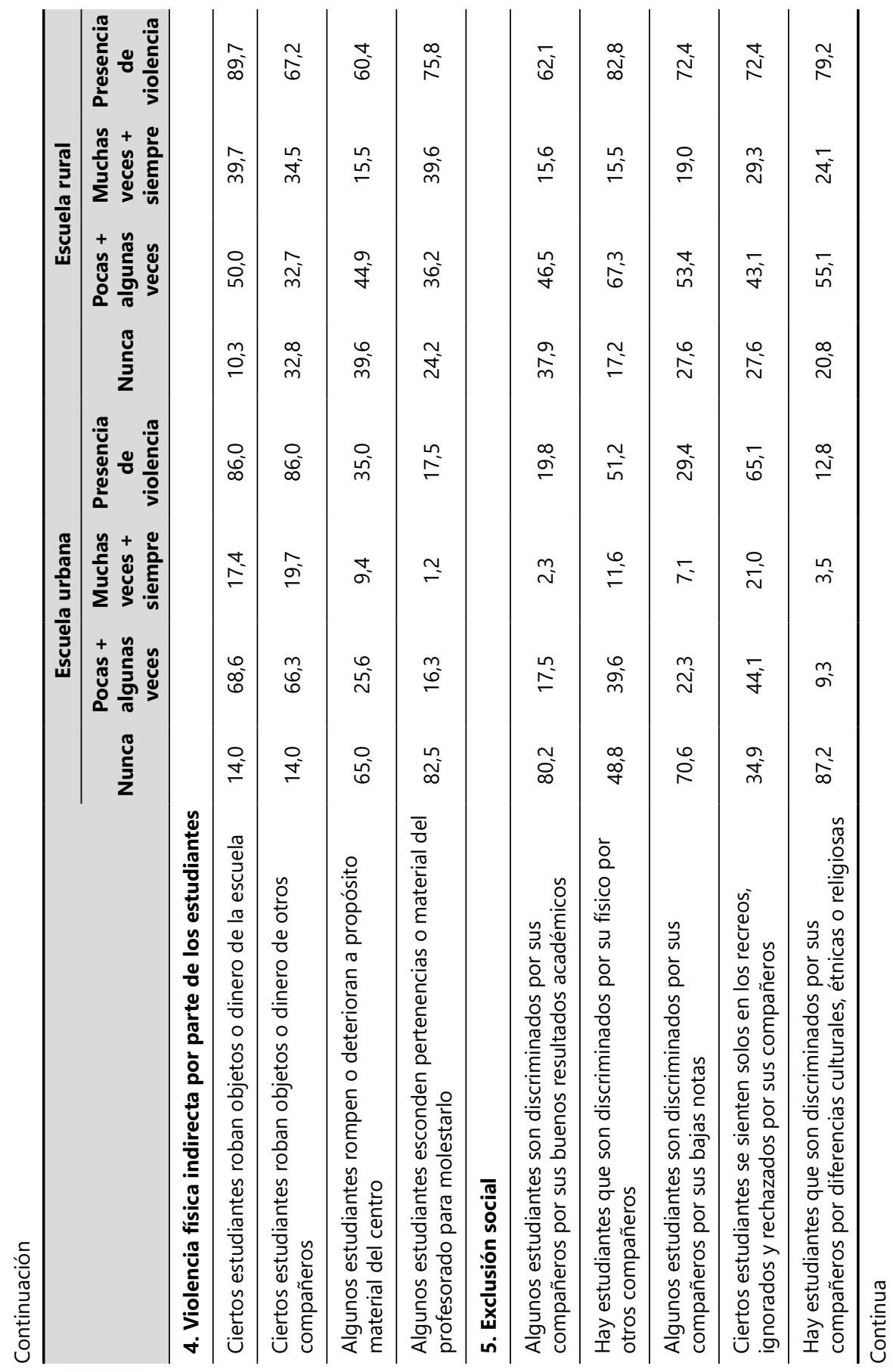




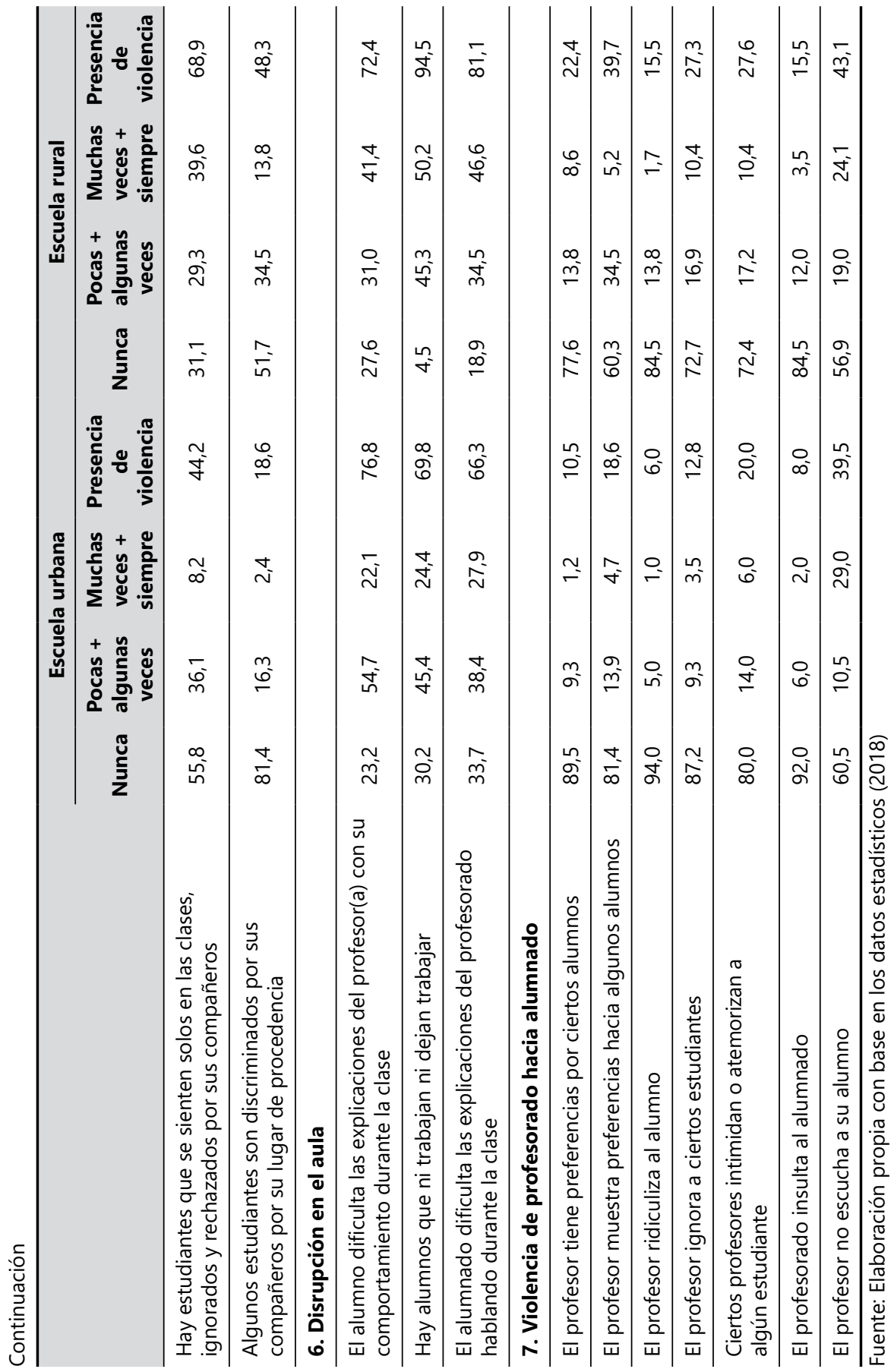


El diagnóstico escolar muestra que de los siete factores considerados en todos hay más presencia de violencia en la escuela rural que en la urbana. De la misma manera en relación a los indicadores, de 34 totales en 30 hay más presencia de violencia en la escuela rural que en la urbana. Los factores en los que se identificó mayor presencia de violencia en la escuela urbana son los relativos a la violencia verbal entre alumnos, seguido de la disrupción en el aula, donde sumados los de baja intensidad y los de alta (desde pocas veces hasta siempre) son mayoría. En el caso de la escuela rural, la mayor presencia de violencia se registró en los mismos indicadores pero de manera inversa: inicialmente, sobre la disrupción en el aula, y después, sobre la violencia verbal entre alumnos.

Los aspectos donde se registró menor presencia de violencia en ambas escuelas fueron los relativos a la violencia del profesor hacia el alumno; sin embargo, el promedio de los indicadores donde no hay presencia de violencia en la escuela urbana es del $83,0 \%$, mientras que en el caso de la escuela rural es de solo el $72,0 \%$; es decir, en más de una cuarta parte el propio profesor en la escuela rural es el que genera violencia en el aula porque la dirige hacia los alumnos. Un segundo aspecto donde se presentó menos violencia fue el relativo a la violencia verbal de los alumnos hacia los profesores; empero, los datos son contrastantes, ya que en la escuela urbana fue en el $84,0 \%$ de los casos donde no se registró violencia; en el caso de la escuela rural, el porcentaje fue del $58,0 \%$ del promedio entre los cuatro indicadores, es decir, en casi la mitad de casos se presentan conductas violentas de baja a alta intensidad por parte de los alumnos hacia sus profesores.

En cuanto a indicadores por separado, los que mayor registro de violencia presentaron fueron los relativos al robo de objetos entre alumnos, donde los porcentajes de violencia acumulan el $89,0 \%$ y $86,0 \%$ respectivamente en la escuela rural y urbana, además del relativo a las expresiones negativas entre alumnos, que sumó el $88,0 \%$ y $81,0 \%$ en el mismo orden, rural y urbana.

En cuanto a los resultados de presencia de violencia entre una y otra escuela, se encontró que de los cuatro indicadores que comprende el primer aspecto, en tres de ellos los porcentajes son muy parecidos en la escuela urbana y rural. Solo en el primero, que se refiere a poner apodos a sus compañeros, la presencia de violencia es mayor en la escuela urbana. Sobre el cuarto indicador, que se refiere a los insultos, es donde existe mayor diferencia entre un centro escolar y otro, siendo mucho mayor en el rural. Sobre el segundo aspecto, que comprende cuatro indicadores que se refieren a la conducta del alumno hacia 
al profesor, la presencia de violencia es diferenciada, ya que en el caso de la escuela urbana la gran mayoría nunca presenta desafíos, retos o insultos a los profesores; sin embargo, el caso de la escuela rural registra más de la mitad de casos de falta de respeto a los docentes; de la misma manera, los desafíos al profesor suman casi el 50,0\%.

De los cinco indicadores del tercer factor referente a la violencia física directa y amenazas entre estudiantes, solo en uno se presenta mayor violencia en la escuela urbana y es prácticamente insignificante ( $0,2 \%$ superior), y es el relativo a dar golpes a compañeros en forma de broma; en los cuatro indicadores restantes la escuela rural supera la presencia de violencia a la urbana, siendo significativos dos de ellos donde los porcentajes suman más del doble en la presencia de violencia. En cuanto a la violencia física indirecta, se describen cuatro indicadores; en tres de ellos la presencia de violencia es superior en la escuela rural que en la urbana; solo en el indicador relativo al robo de objetos a compañeros es donde la escuela urbana supera el porcentaje de la rural. Sin embargo, hay contraste en la presencia de violencia sobre el deterioro que causan a la escuela, ya que en la rural el porcentaje de alumnos que provocan daños casi duplica al de la escuela urbana; y en cuanto a la relación con el profesorado, en el porcentaje referente a ocultarle cosas o molestarlo, la escuela rural registra casi cuatro veces más la presencia de esas conductas.

El quinto factor, referente a la exclusión social, contiene siete indicadores; en todos ellos se registró mayor presencia de violencia en la escuela rural que en la urbana. Curiosamente, los porcentajes que son más contrastantes y se acumulan más en la escuela rural son los relacionados al desempeño escolar y a condiciones físicas o religiosas de los alumnos; en esa escuela, tanto los alumnos que obtienen buenas notas como los que obtienen malas son objetos de violencia. Los indicadores relacionados a la discriminación por el aspecto físico y rechazo entre alumnos en clase o en el recreo también presentan mayores niveles de violencia en la escuela rural que en la urbana, aunque son menos contrastantes.

El factor relacionado a la disrupción en el aula solo comprende tres indicadores; de ellos, en dos se registró mayor presencia de violencia en la escuela rural. Solo el relativo al comportamiento de los alumnos hacia sus maestros en permitir $u$ obstaculizar la impartición de clase es superior en la escuela urbana; sin embargo, casi la totalidad de los alumnos de la escuela rural mostraron conductas que no dejan trabajar al docente; tampoco ellos trabajan (el 94.0\% de los cuestionarios registró ese comportamiento). 
Por último, el aspecto relacionado a la violencia de los profesores hacia los alumnos integra siete indicadores; en todos, el porcentaje es superior en la escuela rural. El menos contrastante es el relacionado a la omisión de los maestros por escucharlos; en situación contraria, los que registran mayor diferencia son los dos primeros relativos a la preferencia que el profesor tiene por y hacia algunos alumnos. Esto significa que la exclusión y violencia es generada en parte por los propios docentes al percibir y asumir conductas de preferencia y discriminación hacia unos y otros alumnos.

En el caso de la escuela rural, en 23 de los 34 indicadores la presencia de violencia supera el 50,0\%, y en el caso de la urbana, solo en 14 es mayor. Los comportamientos más contrastantes se identificaron en relación al factor exclusión social y violencia del docente a los alumnos; los más parecidos se identificaron sobre la violencia física y verbal entre alumnos y la disrupción en el aula.

\subsection{Identificación de la implementación de la política educativa}

Los resultados cualitativos muestran contrastes parecidos a los registrados en el diagnóstico de la primera etapa. El contexto como parte de la propia justificación de la investigación refiere a espacios físicos diferenciados, uno que se denomina urbano por ser la mayor aglomeración poblacional del estado de Tlaxcala; sin embargo, se trata de una ciudad pequeña frente a las principales metrópolis de México. En el caso de la segunda escuela, la localidad se encuentra en condiciones eminentemente rurales, alejada de núcleos urbanos y con marginación socioeconómica. En la ciudad se percibe mayor orden vial, social y comercial con vigencia de la normatividad en el espacio público. En el caso del contexto rural, se identificó desorden en los espacios públicos, carencia de normatividad y de manera muy grave grupos delictivos que operan sin ninguna restricción para extraer y robar combustible de los ductos subterráneos cercanos a la localidad propiedad de Petróleos Mexicanos (empresa del estado).

En relación a las Prioridades del Sistema Básico de Mejora, muestra realidades distintas: las más agudas son en relación a los mecanismos de inclusión para evitar el rezago escolar; en la urbana no solo se incluye a los alumnos que presentan alguna discapacidad, sino que se acepta a alumnos de otras escuelas para ser atendidos e incluidos por personal especializado que ha sido incorporado en el centro escolar. En el caso de la escuela rural, además de no establecer estrategias, los propios docentes son los que generan exclusión y conductas antisociales, a lo que se agregan medidas correctivas como multas ante el incumplimiento 
o inasistencia de los padres de familia a las reuniones, que lejos de orientarse a la prevención, representan ser sancionatorias.

Sobre el horario como escuela de tiempo completo, la escuela urbana registró cumplimiento aunque con cuestionamientos hacia su efectividad por parte de los padres de familia; pero en el caso de la rural, no se cumple, se omite la relevancia del indicador debido a una limitada supervisión por las autoridades del sector educativo. Sobre la Ruta de Mejora Escolar, en ambas escuelas se identificó la carencia de conocimientos para desarrollar un proceso de planeación para incorporar los problemas diagnosticados y su posible atención. La diferencia es que en la escuela urbana los docentes mostraron esfuerzo por llevarla a cabo; en el caso de la escuela rural, lo más grave fue que el propio director desconocía las acciones que deberían instrumentar con base en las orientaciones institucionales.

La organización del Consejo Técnico Escolar es la muestra más objetiva de cómo funciona una escuela, ya que es donde de manera colectiva los docentes y directivos analizan las principales problemáticas para su atención. En el caso de la escuela urbana, se identificó un trabajo metódico y sistemático para el cumplimiento de la programación, seguimiento y registro de las acciones establecidas en la Ruta de Mejora por cuatro comisiones que representan las prioridades del Sistema Básico de Mejora. Aunado a ello, consideraron las sesiones como el auténtico órgano colegiado de trabajo. En el caso de la escuela rural, la ausencia de liderazgo de quien preside el consejo y la apatía de los docentes evidenció la poca importancia que le conceden al trabajo colegiado, y allí además se carece de un orden, registro y seguimiento.

Referente al uso de materiales para la convivencia escolar, en la escuela urbana la respuesta fue que no contaban con ellos; sin embargo, mostraron acciones para atender problemas sobre violencia escolar y otros temas a partir de las sesiones y decisiones de colegiado, las cuales fueron implementadas y revisadas mediante un seguimiento sistemático. Situación contraria se identificó en la escuela rural, donde sí contaban con los materiales distribuidos por la SEP, pero no los utilizaron ni mostraron interés por emplearlos como documentos de apoyo.

\section{Conclusiones}

Como toda política pública, el éxito de sus objetivos depende del adecuado desempeño de cada una de las etapas, que comprende desde cómo se incorpora como asunto público, cómo se diseña, cómo se implementa y cómo se evalúa. 
En el caso de la política educativa de convivencia escolar, al igual que otras estrategias y programas en México, ha sido permanente el cuestionamiento sobre la pertinencia de estandarizar y homologar su diseño cuando las regiones y localidades del país son diferentes y en algunos casos contrastantes social y culturalmente; sin embargo, la dificultad de diferenciar el propio diseño ha conducido a que sea en el proceso de implementación donde los actores adapten los lineamientos para el cumplimiento de los objetivos.

De esta manera, la implementación de la política educativa se revisó en dos escuelas en contextos diferenciados que permitieron diagnosticar el nivel de violencia y las acciones que se han emprendido para su atención. De los resultados obtenidos, se resume que existe mayor presencia de violencia en la escuela rural que en la urbana, y que las estrategias de atención y el trabajo en conjunto que establece la política educativa para los docentes y directivos en la escuela urbana se están instrumentando estrategias que posiblemente han incidido en resultado que arroja el propio diagnóstico, mientras que en la escuela rural no se identificaron avances ni atención por parte de los actores educativos. La afirmación anterior tiene sustento en cada uno de los indicadores cuantitativos y cualitativos obtenidos; la presencia de mayor violencia en varios indicadores es reflejo del contexto familiar, social y cultural.

Resultaría apresurado señalar que a cuatro años de haberse emprendido la política educativa denominada Sistema Básico de Mejora, que en una de sus cuatro líneas promueve la convivencia escolar, se encuentren escuelas completamente transformadas en espacios sanos y pacíficos. Empero, la escuela urbana demuestra que el cumplimiento de los lineamientos institucionales contribuye al mejoramiento de los ambientes escolares. Resulta un tanto aventurado también señalar que a partir de la instrumentación de esta política educativa se generaron los cambios, ya que no se cuenta con antecedentes previos a la reforma sobre el comportamiento específico de estas dos escuelas, como de muchas otras, y ya que las conductas posiblemente son las mismas que antes de la política educativa de convivencia escolar; lo que sí se pudo identificar es que prevalece una relación estrecha entre la situación de violencia que registró el diagnóstico y las acciones que directivos y docentes han estado y están instrumentando en relación a cada uno de los programas y estrategias de política educativa.

De manera particular sobre los programas implementados, se identificó que en la Ruta de Mejora Escolar, la implementación de una adecuada planeación 
determina los resultados; en el caso de la escuela rural al no existir, los resultados son diferentes. En relación a las Prioridades del Sistema Básico de Mejora, la escuela urbana, además de contar con los especialistas para la atención de alumnos con discapacidad, promueve la inclusión y convivencia pacífica. En cuanto al funcionamiento de la escuela de tiempo completo, si bien se identificaron algunas objeciones en la escuela urbana, hay cumplimiento de horarios y actividades; de esa condición la escuela rural carece. El Consejo Técnico Escolar en la escuela urbana opera con el liderazgo de la directora; por el contrario, en la escuela rural fue evidente la indiferencia. Por último, sobre los materiales educativos, en la escuela urbana se difunden parcialmente y la rural, si bien cuenta con ellos, no los instrumenta.

En resumen, la política educativa para la convivencia escolar considera a la etapa de implementación como la que define los resultados, porque es el momento en que se materializan las acciones. El diseño de cada una de las estrategias de la política educativa a través de los programas institucionales revisados parece ser acertada, pero su éxito depende completamente de su implementación y seguimiento. 


\title{
Educational policy, violence and school coexistence. The experience in two schools
}

\begin{abstract}
Before the slightly effective results that the corrective approach showed to take charge of violence, some prevention-oriented proposals appeared, demonstrating that it is better anticipating than correcting antisocial conducts. In that frame, school harmony has been chosen as a tool in the academic and institutional fields in Mexico. This research's objective was to identify the development of the educational policy oriented towards fostering school coexistence in two primary schools with different contexts in the state of Tlaxcala, Mexico. The working method consisted of two phases: 1) the diagnosis of the school violence situation and 2) the identification of the school harmony policy implementation. The results show violence situations and strategies relatively contrast.
\end{abstract}

Keywords: Educational policy. School coexistence. School. Inclusion.

\section{Política educacional, violência e convivencia escolar. A experiência em duas escolas}

\section{Resumo}

Diante dos resultados ineficazes demonstrados pela abordagem corretiva para enfrentar o fenômeno da violência escolar, surgiram propostas orientadas à prevenção, demostrando que é melhor prevenir as condutas antissociais do que corrigi-las. Nesse contexto, a questão da convivência escolar foi registrada como uma ferramenta de trabalho bem recebida pela academia e pelas instituições no México. Dessa maneira, o objetivo da pesquisa foi identificar a política educacional orientada ao desenvolvimento da convivência em duas escolas com contextos diferentes da Educação Básica do Estado de Tlaxcala, México. O método de trabalho consistiu em duas etapas: 1) diagnóstico da situação de violência escolar e 2) a identificação da implementação de uma política para convivência escolar. Os resultados demonstraram situações de violência e distintas estratégias implementadas onde os docentes e os diretores são identificados como atores vertebrais da politica educacional proposta.

Palavras-chave: Politica educacional. Convivência escolar. Escola. Inclusão. 


\section{Referencias}

ÁLVAREZ-GARCÍA, D.; NÚÑEZ, J.C.; DOBARRO, A. Cuve-EP: cuestionario de violencia escolar - 3: manual de referencia. Bizkaia: Grupo ALBOR-COHS, 2015.

ARARTEKO. Convivencia y conflicto en los centros educativos. Vitoria-Gasteiz: Ararteko. 2006.

ARON, A. M.; MILICIC, N. Clima social escolar. México: Trillas; 2013.

CASTRO-PÉREZ, M.; MORALES-RAMIREZ, M. E. Los ambientes de aula que promueven el aprendizaje, desde la perspectiva de los niños y niñas escolares. Revista Electrónica Educare, Heredia, v. 19, n. 3, p. 1-32, set. 2015. https://doi.org/10.15359/ree.19-3.11

COLOMBO, G. B. Violencia escolar y convivencia escolar: descubriendo estrategias en la vida cotidiana escolar. Revista Argentina de Sociología, Buenos Aires, v. 8-9, n. 15-6, p. 81-104, 2011. Disponible en: http://www.redalyc.org/articulo.oa?id=26922386005. Acesso en: 2 marzo 2018.

DE LA CONCEPCIÓN, A. Concepciones sobre participación de niñas, niños y adolescentes: Su importancia en la construcción de la convivencia escolar. Cultura Educación y Sociedad, [s. l.], v. 6, n. 2, p. 9-28, 2015. Disponible en: https://revistascientificas.cuc.edu.co/culturaeducacionysociedad/article/ view/840. Acceso en: 10 abril 2018.

FIERRO, C.; CARBAJAL, P.; MARTÍNEZ-PARENTE, R. Ojos que sí ven: Casos para reflexionar sobre la convivencia escolar. México, DF: Ultra, 2014.

FIERRO, C. et al. Convivencia escolar: un tema emergente de investigación educativa en México. In: FURLÁN, A.; T. C. SPITZER, T. C. (coords.). Violencia en las escuelas: problemas de convivencia y disciplina. México: Comie-Anuies, 2013. p. 71-132.

FUNK, W. Violencia escolar en Alemania: estado del art. Revista de Educación, [s. l.], n. 313, p. 53-78, 1997. Disponible en: http://www. sinohacesnadasosparte.org/Download/spanish/04_ESTADO_ARTE_ VIOLENCIA_ESCOLAR_ALEMANIA.pdf. Acceso en: 12 marzo 2018.

FURLÁN, A.; SAUCEDO, C.; LARA, B. (coords.). Miradas diversas sobre la disciplina y la violencia en centros escolares. Guadalajara: Universidad de Guadalajara, 2004. 
GIRALDO, L.; MERA, R. Clima social escolar: percepción del estudiante. Colombia Médica, Cali, v. 31, n. 1, p. 23-27, 2000. Disponible en: http://www. redalyc.org/html/283/28331106/. Acceso en: 07 mayo 2018.

HIRMAS, C.; EROLES, D. (coords.). Convivencia democrática, inclusión y cultura de paz: Lecciones desde la práctica educativa innovadora en América Latina. Santiago de Chile: OREALC, 2008.

HUBERMAN, A. M.; Miles, M. B. Data management and analysis methods. In: Denzin, N. K.; Lincoln, Y. S. (eds.). Handbook of qualitative research. Thousand Oaks: CA: Sage, 1994. p. 428-444.

JARES, X. Educación para la paz. Madrid: Editorial Popular, 1999.

LIRA, A.; GOMES, C. A. Violence in schools: what are the lessons for teacher education?. Ensaio: Avaliação e Políticas Públicas em Educação, Rio de Janeiro, v. 26, n. 100, p. 759-779, jul/set. 2018. https://doi.org/10.1590/s0104-40362018002601574

MÉXICO. Acuerdo $n^{\circ} 703$ por el que se emiten las reglas de Operación del Programa Escuelas de Calidad. México. Diario Oficial de la Federación, México, DF, 28 dez. 2013a.

MÉXICO. Acuerdo $\mathrm{n}^{\circ} 717$. Por el que se emiten los lineamientos para formular programas de gestión escolar. Diario Oficial de la Federación, México, DF, 7 mar. 2014b.

MÉXICO. Decreto. Decreto por el que se reforman los artículos 3o, 5o, 24, 27,130 y se adiciona el artículo decimoséptimo transitorio de la constitución política de los Estados Unidos Mexicanos. Diario Oficial de la Federación, México, DF, 28 jan. 1992.

MÉXICO. Decreto. Decreto por el que se reforma la fracción VI del artículo 7 de la Ley General de Educación. Diario Oficial de la Federación, México, DF, 17 jun. 2008.

MÉXICO. Decreto. Decreto por el que se expide la Ley General para la Prevención Social de la Violencia y la Delincuencia. Diario Oficial de la Federación, México, DF, 24 jan. 2012b.

MÉXICO. Lei general de Educación. Diario Oficial de la Federación, México, DF, 30 set. 2019. 
MÉXICO. Gobierno de la Republica. Plan nacional de desarrollo. México, DF: SEGOB, 2013b.

MÉXICO. Secretaría de Educación Pública. Lineamientos para la organización y funcionamiento de los Consejos Técnicos Escolares de Educación Básica. México, DF, 2017.

MÉXICO. Secretaría de Educación Pública. Programa de escuelas de tiempo completo. México, DF, 2012a.

MÉXICO. Secretaría de Educación Pública. Programa Nacional de Convivencia Escolar. México, DF, 2016.

MÉXICO. Secretaría de Educación Pública. Proyecto a favor de la Convivencia Escolar (Pace): ropuesta educativa para favorecer la convivencia en las escuelas. México, DF, 2014c.

MÉXICO. Secretaría de Educación Pública. ¿Qué es la Escuela de Tiempo Completo?. México, DF, 2013c.

MILCÍADES, V. La formación para la convivencia: un reto para la gestión escolar. Espacio Abierto, [s. l.], v. 24, n. 3, p. 118-119, jul.-set. 2015. Disponible en: http:// www.redalyc.org/articulo.oa?id=12242627006. Acceso en: 15 abril 2018.

ORGANIZACIÓN DE LA NACIONES UNIDAS - ONU. Declaración Universal de Derechos Humanos. New York, 2018.

RADA, D. M. El rigor en la investigación cualitativa: técnicas de análisis, credibilidad, transferibilidad y confirmabilidad. Revista Venezolana de Investigación, [s. l.], v. 7, n. 1, p. 17-26, 2007.

RODNEY, Y.; GARCÍA, M. Estudio histórico de la violencia escolar. Revista Científica-Metodológica Varona, [s. l.], n. 59, p. 41-49, 2014. Disponible en: http://www.redalyc.org/articulo.oa?id=360636905008. Acceso en: 27 marzo 2018.

SANDOVAL MANRÍQUEZ, M. Convivencia y clima escolar: claves de la gestión del conocimiento. Última Década, [s. l.], v. 22, n. 41, p. 153-178, dic. 2014. https://doi.org/10.4067/S0718-22362014000200007

SAUCEDO, C. L.; MEJÍA, J. M. G. ¿Es posible la convivencia escolar? Aportes analíticos desde la realidad de la escuela en nuestros días. In: MARTÍNEZ RUIZ, X.; ROSADO MORENO, D. (coords.). Estudios 
de la juventud y filosofía de la no violencia: conciencia generacional, ciudadanía y argumentación México, DF: Quinta del Agua, 2013. p. 159184.

\section{Información del autores}

Adriana Carro Olvera: Doctora en Desarrollo Regional. Investigadora de la División de Estudios de Posgrado por la Facultad de Ciencias de la Educación de la Universidad Autónoma de Tlaxcala. Miembro del Sistema Nacional de Investigadores Nivel I y socia del Consejo Mexicano de Investigación Educativa. Contacto: acarroo1@yahoo.com.mx

iD http://orcid.org/0000-0001-6086-9152

José Alfonso Lima Gutiérrez: Doctor en Desarrollo Regional por el Colegio de Tlaxcala Asociación Civil. Docente Investigador de la Universidad Autónoma de Tlaxcala con Perfil Deseable del Programa de Desarrollo del Profesorado. Contacto: alimagu_1@yahoo.com.mx

iD http://orcid.org/0000-0002-7910-9003 